Fig. 2. Stück einer Alveole von einer mit Chlorsilber injicirten Kalbslunge. Vergrösserung 600 .

Fig. 3. Alveole einer Hammellunge, deren Bronchien mit ungefärbten Leimmassen injicirt sind. Vergr. 500. a Epithel der Alveolen im Profil. b Dasselbe von der Fläche. c Bindegewebskerne. d Gefässschlingen. e Capiflarkerne. f Begrenzendes Alreolengewebe. Elastische Fasern der Bläschenwand.

Fig. 4. Proflansicht einer Alveole, deren Gefässe mit Chromblei injicirt sind. Vergr, 600. a Gefässschlingen. b Bläschenwand. c Epithelien an dem theilweise noch sichtbaren Bläschengrund. d Bindegewebskerne. e Epithel in Profilansicht. f Gefässschlingen, die in das Alveolenlumen bineinragen.

\title{
XXII.
}

\section{Histologisches Detail zu der grauen Degeneration von Gehirn und Rückenmark. (Zugleich ein Beitrag zu der Lelire von der Entstehung und Verwandlung der Zelle.)}

\author{
Von Dr. Eduard Rindfleiseh, \\ pathologischom Prosector in Zürich.
}

(Hierzu Taf. XI. Fig. 5-7.)

Unter der Symptomengruppe der sogenannten Tabes dorsalis war um Pfingsten dieses Jahres im biesigen alten Spital ein Mann gestorben; bei der Section ergab sich, dass Gebirn und Rückenmark im hohen Maasse jene Form chronischer Entzündung darboten, welche am häufgsten mit dem Namen der grauen Degeneration bezeichnet wird. In den Vordersträngen des Rückenmarkes, am Fornix, Corpus callosum, Centrum Vieussenii war die Continuität des Marklagers durch eine grosse Anzahl hirsekorn- bis erbsengrosser, meist länglicher Heerde unterbrochen, welche die verschiedensten Stadien des fraglichen Prozesses, von dem Zustande der überwallenden, grauröthlichen Pulpa bis zu der lückenartig erscheinenden, grauen Schwiele darboten. Nach Rokitansky handelt es sich hierbei um eine heerdweise Vermebrung des interstitiellen Bindegewebes. Die Neubildung habe anfangs den Cha- 
rakter , der durch eine formlose Grundsubstanz und durch die zahlreichen schimmernden Kerne ausgezeichneten Ependymformation" und gehe später in die faserige Narbensubstanz über. Sie verdränge und zerstöre die Nervenfasern und schliesse zu allen Zeiten Fettkömchenzellen und Amyloidkörper ein, welche als Umwandlungsproducte der Markscheiden anzusehen seien. Ich habe das obenerwähnte Gehirn und Rilckenmark sowohl frisch als nach Erhärtung in Chromsäure, Carminfärbung etc. untersucht, und muss Rokitansky's Auffassung in einem wesentlichen Punkte bestätigen. Wir haben es in der That mit einer Metamorphose der Bindesubstanz zu thun, welche wir als eine Neubjldung bezeichnen können. Im einzelnen aber sind meine Resultate so abweichend von denen Rokitansky's, dass ich es für zweckmässig erachte, eine eigene, zusammenhängende Darstellung des auatomischen Verlaufes zu geben.

Wenn man die frischveränderten Partien im Marklager des Gehirnes aufmerksam betrachtet, so gewahrt man schon mit blossem Auge in der Mitte jedes einzelnen Heerdes einen rothen Punkt oder Strich, das quer oder schräg durchschnittene Lumen eines mit Blut überfüllten Gefässstämmchens; am Rückenmarke stellen sich die ersten Verïnderungen so dar, dass die grauen Heerde (auf dem Querschnitt) keilförmig von der Peripherie, namentlich aber dem Sulcus longitudinalis anterior in die Substanz der Vorderstränge eingreifen; Form und Lage derselben entspricht genau dem Verbreitungsbezirke je eines der kleinen Gefässe, welche von der Pia mater aus in das Rückenmark eintreten und auch bier als rothe Striche oder Punkte in dem Heerde erscheinen. Alles führt uns darauf bin, den ersten Grund der Erkrankung in einer Veränderung einzelner Gefässe und ihrer Verzweigungen zu suchen; eine Vermuthung, welche von der mikroskopischen Untersuchung vollständig bestätigt wird. Sämmtliche Gefässe, welche innerhalb der Heerde verlaufen, aber auch diejenigen, welche das nächstumgebende, noch intacte Parenchym durchziehen, befinden sich in einem Zustande, welcher für chronisch-entzündliche Prozesse charakteristisch ist (Vergl. Virchow's Beobachtungen an der Innenfläche hydropischer Ventrikel, Billroth, am entzulnd. Präputium). Itre 
Wandungen sind durch eine Anbäufung von Kernen und Zellen in der Adventitia enorm verdickt. Während man im normalen $\mathrm{Zu}$ stande an dieser Membran nur hie und da einen einzelnen kern wahrnimmt, besteht sie jetzt aus einer oft fünffachen Lage libereinander geschichteter Elemente. Dabei ist das Lumen der Gefässe nicht etwa verengt, sondern beträchtlich dilatirt, was zum Theil aut eine Alteration der Media zu beziehen ist. Ich habe mich an derartigen Gefässen mehrmals überzcugen können, dass die glatten Muskelfasern dieser Membran nicht mehr in der charakteristischen Gestalt und Anordnung vorhanden waren. Auch an den Capillaren waren Veränderungen bemerklich; dass man an den Wandungen der Capillargefässe doppelte Contouren wahrnimmt, ist nicht merkwürdig; aber wenn diese Contouren stellenweise so weit auseinanderrücken, dass eine Lage rundlicher Zellen zwischen ihnen Platz hat, welche augenscheiulich von den Kernen des Capillargefiisses abstammen, so sind dies Bilder, welche sich mit unseren gegenwärtigen Vorstellungen von dem Bau der Capillargefässwände nur schwer vereinigen lassen. Entweder hatten diese Capillargefässe von vornherein doppelte Wandungen, zwischen denen die Kerne eingebettet waren, oder es handelt sich hier um eine enorme Verdickung und Aufquellung jener einfachen Wandung, welche man ihnen allgemein zuerkennt.

Die beschriebenen Veränderungen einzelner Gefässbäumchen bilden nach meinem Dafürhalten das erste Glied des anatomisch nachweis,baren Prozesses. Ich erblicke darin das Resultat hälıfig wiederkehrender oder lange anhaltender, mit Hyperämie verbundener Reizzustände der gesammten Centralorgane. Dass dieselben nur an einzelnen Abschnitten des Gefässsystems bleibende Spuren zurücklassen, geschieht im Einklang mit fast allen diffusen 0ryanerkrankungen, die sich, genau betrachtet, immer auf eine, wenn auch noch so grosse Anzahl einzelner Heerde zurückfuhren lassen. Es bestehen aber diese bleibenden spuren in einer formativen Reizung, welche sich zunächst in den Wandungen der Gefässe selbst einstellt, danach aber auf die anstossende Neuroglia übergeht. Die Neuroglia macht in Folge dessen eine Reihe von Meta morphosen durch, welche für dic Pathologie dieser Substanz, so 
wie fär die Lehre von den Neubildungen tberhaupt nnd denen des Gehirnes insbesondere von grossem Interesse sind.

Zunächst ist die Frage noch mit nichten entschieden, welche von den mikroskopisch darstellbaren Elementartheilen des Gehirns und Rückenmarks man als Bindesubstanz anzusehen habe. Die Ansichten der Autoren gehen daruber soweit auseinander, dass zum Beispiel Jacubowitseh gar keine, Bidder und seine Schuler eine grosse Menge annehmen und gradezu alles für Bindesubstanz erklären, was nicht deutlich die Charaktere der Ganglienzellen oder der Nervenfasern hat. Von dem Votum der pathologischen Anatomie muss in dieser Beziehung deshalb sehr viel abhängen, weil dieselbe anderweitig die Thatsache constatirt hat, dass gewisse Entwicklungen nur vom Bindegewebe ausgehen und darum eine wohlberechtigte Vermuthung vorliegt, dass, wenn man im Gehirn und Rückènmark denselben Entwichlungen begegnet, dann diejenige Substanz als Bindegewebe zu betrachten sein dürfte, von welcher sie ausgehen. Ich hatte in dieser Richtung bereits einige Beobachtungen gemacht, die mir nummehr zu Statten kamen. Dieselben beziehen sich auf die crste Entstehung und das Wachsthum jener solitüren, käsigen Knoten, wolche man wohl mit Unrecht als wahre Tuberculose des Gehirns bezeichnet. Es handelt sich dabei nur um eine ausschreitende Vermehrung und soforlige Verkäsung der als „Körner" bekannten Elementartheile des Gehims. Dieselben bilden cine zusammenhängende lage von namhafter Dicke in der inneren Schicht der Kleinhirnrinde, eine minder zusammenhängende in der inneren Schicht der Grosshirnrinde und lassen sich von hier aus in einer gleichmässigen, wenn auch sparsamen Vertheilung durch das Narklager hin verfolgen, bis sie an der Oberfläche der Ventrikel als Kerne des Ependyms wieder elwas reichlicher werden. Schon der gewöhnliche Silz der solitären Tuberkel, nämlich gerade die innere lage der grauen Substanz am grossen wie am klinen Hirn, lässt uns an die Körner denken. Das Mikroskop zeigt an der Grenze der käsigen lleerde unzählige kerne und kleine Zellen, die ich zum Theil freilich nicht von Körnern unterscheider, aber doch auch nicht ohne weiteres für identisch mit ilnen etklären konnte. Dazu bedurfte es einer weiteren Bestätigung. Jede 
Ganglienzelle des Gehirns und Rilckenmarks ist im normalen $\mathrm{Zu}$ stande von allen Seiten in die bekannte feingranulirte Grundsubstanz eingehallt, welche sich bei der Erhärtung in Cbromsäure dergestalt von ihr zuruckzieht, dass eine rundliche, relativ grosse Lücke entsteht, aus welcher (an einem feinen Segmente) die Canglienzelle herausfallen würde, wenn sie nicht durch ihre Fortsätze festgehalten würde. Der Rand dieser Lücke zeigt meistens an einer Stelle ein charakteristisches "Korn" eingebettet. Iśt nun der oben erwähnte Wucherungsprozess an eine solche Stelle herangetreten, so sieht man statt des einen Korns eine grössere und endlich eine grosse Anzahl von Kernen und kleinen Zellen einen Ring bildend, innerbalb dessen die Ganglienzelle in fettiger Entartung begriffen sichtbar ist.

Nach diesem war mir die Bedeutung der Körner als Kerne der Neuroglia im höchsten Grade wahrscheinlich. Durch die gegenwärtige Untersuchung glaube ich sie endlich zur Evidenz bringen zu können. - Ich war dahin gelangt, die Fortselzung der formativen Reizung von den Gefässwänden auf die Nachbarschaft zu schildern. Dieser Prozess trä̀gt durchaus den Stempel des Kümmerlichen. Um so leichter ist es, seine verschiedenen Stadien an hinlänglich feinen Schnitten zu verfolgen. Die Uebersichtlichkeit der Bilder wird durch keine üppige Zellenwucherung gestört, vielmehr gewahrt man selbst an der Grenze des Heerdes, also da, wo man die ersten Stadien der Veränderung zu suchen hat, nur eine sehr mässige Vermehrung der Körner, welche, wie jch oben bemerkte, in regelmässigen Abständen zwischen den Nervenfasern vertheilt sind. Wie man an der Verknöcherungsgrenze des Hyalinenknorpels alle Uebergänge von einer einzelnen Knorpelzelle zu einzelnen Gruppen von mehreren wahrnimmt, so sieht man hier ein Korn, dann zwei Körner, drei oder mehrere Körner zu kleinen Reihen geordnet und so der Länge nach zwischen den Nervenfasern eingebettet. Zugleich geht eine Veränderung in der Grundsubstanz vor sich. Zieht man die bier noch ganz intacten Nervenfasern auseinander, so spannen sich zwischen ihnen äusserst feine Fasern aus, welche auch an den Schnittenden hervorragen. Dieselben verhalten sich chemisch und physikalisch wie elastisches 
Gewebe; sie sind auf längere Strecken gewöhnlich zickzackförmig oder spiralig gebogen und leisten den üblichen Reagentien Widerstand. lch kann nicht anders glauben, als dass sie aus der sonst amorphen Grundsubstanz der Neuroglia hervorgegangen sind. Uebrigens bewirkt das Mehr von Elementarbestandtheilen und die grössere Consistenz, welche die faserige vor der amorphen Grundsubstanz voraus hat, dass sich die Grenze des Heerdes auf dem Durchschnitt als eine kleine, nur bei spiegelndem Lichte auffallende Anschwellung darstellt, welche indessen nach innen zu sofort wiederum abfällt und sogar bis unter das frühere Niveau einsinkt, wenn nicht ein seitlicher Druck ausgeubt wird.

Der Grund dieser letzten Erscheinung ist in der eintretenden Atrophie der Nervenfasern zu suchen, welche sich als ein selbstständiges Glied in der Reihe der Erkrankungen an das eben geschilderte Stadium der Bindegewebsentwicklung anschliesst. Ich komme noch einmal auf den Grund dieses in symptomatischer Beziehung so wichtigen Phänomens zurück und erwähne hier nur, dass mikroskopisch die Nervenfasern zunächst ihr Mark verlieren und dann als dünne, aus Axencylinder und Scheide oder vielleicht nur aus dem Axencylinder bestehende Fäden in das Bindegewebe des Heerdes eine Strecke weit verfolgt werden können. Ausnahmsweise habe ich sie mit einem Marktropfen endigen sehen, in welchem das Ende des Axencylinders aufyerolt erschien. Freie Marktropfen waren immer nur in frischen, niemals in erhärteten Präparaten aufzufinden, obglejch sich der Markstoff in Chromsäure vollkommen erbält. Ich schliesse daraus, dass sie überhaupt durch die Präparationsmethode freigemacht werden, wie dies ja durch Abstreichen und Zerzupfen so leicht geschieht. Jedenfalls werde jch in der Folge zeigen, dass es nicht diese vielgestaltigen Tropfen sind, welche die fettige oder amyloide Metamorphose durchmachen und als Körnchenkugeln und Amyloidkörper im grauen Heerde gefunden werden.

Fahren wir nun in der Betrachtung der Bindegewebsmetamorphosen fort. In dem Masse, als die Nervenfasern weniger Raum einnehmen und sich endlich ganz verlieren, tritt das Bindegewebe mehr hervor. Die feinen Fasern bilden einen dichten, elastischen 
Filz; in diesem erblickt man eine grosse Anzahl einzelner Kerne und einkerniger Zellen neben jeñen kleinen Gruppen von Kernen, welche wir aus der Theilung eines einzelnen Kornes hervorgeben sahen, und welche zum Tbeil an einer länglichen und eingeschnürten Gestalt erkennen lassen, daśs sich der Theilungsvorgang an ihnen noch immer" wiederholt. Und jetzt erfolgt eine interessante Epscheinung; die Entstehung der in der beigegebenen Figur abgebildeten Körper. Es sammelt sich um diese zu Gruppen vereinigten Kerne eine gewisse Menge ganz feingranulirter, das Licht sehr schwach brechender Substanzen, wodurch unregelmässig begrenzte, grössere, zellenartige Plaques entstehen, ähnlich den von Robin und Kölliker beschricbenen, vielkernigen Elementen a us dem Knochenmark.

Die Kerne liegen alle exeentrisch, längs der äussern Begrenzungslinie der Plaque, was den Anschein erweckt, als seien sie durch die protoplasmaartige Substanz auseinandergedrängt. Zerzupft man einen feinen Schnitt, so zeigt sich ein höchst auffallendes Verhältuiss zu der Grundsubstanz; der faserige Filz der letzteren zertheilt sich nämlich dergestalt, dass auf jeden einzelnen der zellenartigen Körper eine gewisse Menge von Fasern kömmt, die von seiner Peripherie, wie ein Wald von Ausläuferm, nach allen Seiten hin abtreten. Diese Formation*) scheint den Höhepunkt der Bindegewebsentwicklung darzustellen, sie ist insofern bemerkenswerth, als sie auf keine Weise in die bekannteren Rahmen der Zellenentwicklung passt. Wenn wir wollen, sehen wir hier Zellen mit Ausläufern vor uns, aber die Zellen sind nicht endogen, nicht durch die Theilung einer präexistirenden Zelle entstanden, sondern dadurch, dass präcxistirende Kerne durch eine Auläufung von Pıtoplasma zu einem abgeschlossenen Körper vereinigt wurden; die Ausläufer aber sind Fasern, wetehe sich vielleicht in organischer Beziebung zu den Kemen cntwickelt haben, die abcr doch wesentlich aus Grundsubstanz hervorgegangen sind (siehe die Schlussbemerkung).

*) Zuerst ron Leubuschel aufgefonden und besclirieben. Virchow's Archiv XIII. 494 . 
Das Ganze ist wie ein Schwamm getränkt von einer schleimigen, in Wasser etwas gerinnenden Flüssigkeit, welche eine gewisse Menge von Kernen und kleinen einkernigen Zellen enthält, die ebenfalls als Abkömmlinge der Neurogliakerne zu betrachten sind. Diese Flüssigkeit tritt an einer frischen Schnittfläche auf seitlichen Druck hervor und man kann durch sie, beilăufig gesagt, leicht zu einer einseitigen Anschauung über die Natur des neugebildeten Bindegewebes kommen. Da nämlich die Fasermasse mit den grossen Zellen eine so zähe, elastische und zusammendrückbare Substạnz bildet, dass sie sich im frischen Zustande gar nicht und selbst nach Chromsäurebehandlung nur sehr schwer in feine Schnitte zerlegen lässt, so bekommt man durch eine einfache Priparationsmethode, namentlich aber durch Abnehmen der oberflächlichsten, überquellenden Schicht immer nur jene ependymartige Formation Rokitansky's zu Gesicht.

Im weiteren Verlaufe des Prozesses stellen sich regressive Metamorphosen ein. Fettkörnchen treten in den Zelien auf und indem sie sich mehren, entstehen jene grossen Fettkörnchenkugeln, welche man schon mit der Lupe an der Schnittfäche eines grauen Heerdes als weisse Pünktchen wahrnimmt. Dass es wirklich Zellen sind, aus welchen die Körnchenkugeln entstehen *), zeigt sich am besten daraus, dass man nach Carminfärbung in jeder derselben einen oder mehrere rothgewordene Kerne nachzuweisen im Stande ist, was sich durchaus nicht mit der Annahme verträgt, dass dieselben, wie Rokitansky will, aus Bruchstiucken der Nerven-Nlarkscheiden hervorgegangen seien. Ebenso verlält es sich meiner Ansicht nach mit den Amyloidkörpern. Ich habe nicht nur keine Veranlassung gefunden, dieselben von Marktropfen abzuleiten, sourdern habe auch bei einer anderen Gelegenbeit eine Beobachtung gemacht, welche mich vielmehr bestimmt anzunehmen, dass die kleinen, einkernigen Zellen der schleimigen Flussigkeit zwischen den Fasern die amyloide Metamorphose durchmachen. Es handelte sich damals um eine Perihepatitis. Eine Froschleber war an mehreren Stellen mit flachen, rundlichen Anhäufungen einer gallertigen

*) Eine Thatsache, die zuerst Virch ow hervorgehoben hat.

Archiv f. pathol. Anat. Bd. XXvi. IIft. 5 u. 6. 
Substanz bedeckt. Auf dem senkrechten. Durchschnitt zeigte die letztere mehrere übereinandergeschichtete homogene Lamellen, welche durch eingeschobene Lagen runder, einkerniger Bindegewebszellen getrennt waren. Unter diesen Bindegewebszellen, welche den in Rede stehenden Elementen der grauen Degeneration glichen, grassirte die amyloide Metamorphose. Sobald man verdünnte Jodlösung hinzubrachte, wurden einige vollkommen jodroth, bei anderen wurde der Kern dunkler als die ubrige Substanz der Zelle, bei noch anderen färbte sich nur der Kern, so dass man alle Uebergänge von ganz normalen Zellen $\mathbf{z u}$ amyloiden Körperchen wahrnehmen konnte. Uebrigens babe ich die Amyloidkörper vorzugsweise in dem letzten Stadium unserer Veränderungen, der narbigen Verdichtung angetroffen.

Die narbige Verdichtung ist ein mechanischer Prozess. Wenn pämlich die graue Degeneration in der beschriebenen Weise diejenigen Grenzen erreicht hat, welche ihr, wahrscheinlich durch die Ausdehnung der Gefässerkrankung, zu erreichen bestimmt sind, und wenn dann die feltige Degeneration die grosse Mehrzahl der Zellen in einen resorptionsfähigen Detritus verwandelt hat, so behalten wir nur die feinen elastischen Fasern zurück. Diese suchen sich zusammenzuziehen und üben auf die in ihren Zwischenräumen enthaltene Flüssigkeit einen Druck aus, welcher ohne Zweifel die Resorption derselben in hohem Grade begünstigt. In dem Maasse aber die Flüssigkeit schwindet, rücken die Fasern dichter und dichter aneinánder, bis sie sich endlich unmittelbar und ohne alle Zwischenräume berühren. So finden wir sie als constituirendes Gewebe der Narben, welche nach dem vollständigen Ablaufe des Prozesses zurückbleiben und dadurch, dass sie ganze Abschnitte der Nervensubstanz des Marklagers der Centralorgane durch eine relativ sehr geringe Quantităt eines höchst unnachgiebigen, derben, trockenen Gewebes ersetzen, zu den grössten Deformitäten Veranlassung geben lönnen.

Soviel ron dem histologischen Detail der grauen Degeneration. Wir müssen, um dies kurz zu recapituliren, drei neben einanderhergehende Reihen von Veränderungen unterscheiden, nämlich erstens die Veränderungen der Gefässe, zweitens die Atrophie der nervösen 
Elemente und drittens die Metamorphose der Bindesubstanz. Durch die Verdickung der Gefässwandungen wird ohne $\mathrm{Z}$ weifel ein bedeutendes Hinderniss in den regelmässigen Gang der Ernährung eingeführt. Hieraus erklärt sich einerseits der atrophische Zerfall der Nervenelemente, welche die Leitung zwischen Centrum und Peripherie unterbricht und somit als die unmittelbare Ursache der verschiedenen paretischen und paralytischen symptome der Rückenmarkschwindsucht angesehen werden muss. Andererseits finden wir es in Anbetracht der schlechten Ernährungsverbältnisse begreiflich, dass der Prozess in Bindegewebe mehr eine langsame Umbildung als eine Neubildung zu nennen ist, obwohl er den Charakter der letzteren in der Kernvermehrung deutlich zur Schau trägt. Dass er die Atrophie der Nervenelemente bedingen sollte, wie Rokitansky will, ist mir in dem vorliegenden Falle nicht wabrscheinlich geworden.

Zum Schluss noch eine theoretische Bemerkung, betreffend den Entstehungsprozess der anbei abgebildeten Zellen. Wenn es erlaubt wäre, die Grundsubstanz der Neuroglia als die verschmolzenen Protoplasmen, die Körner aber als Kerne derjenigen Zellen anzusehen, aus welchen sich das Marklager des Centralnervensystems oder auch nur das Bindegewebe dieses Marklagers gebildet hat, so würden wir in dem erwähnten Prozesse eine WiederScheidung der verschmolzenen Protoplasmen erblicken in der Art, dass das zu je einer Zelle gehörige Protoplasma, indèm es sich um die entsprechenden Kerne oder vielmehr um die daraus hervorgegangenen Kerngruppen sammelt, in der Nähe der Kerne mehr flüssig bleibt, während es an der Peripherie zu den gedachten feinen Fasern erstarrt. Es ist diess meine subjective Ueberzeugung von dem Verhältnisse, in welchem die Faserbildung zur Kernwucherung und zur Ansammlung von Protoplasma steht. 


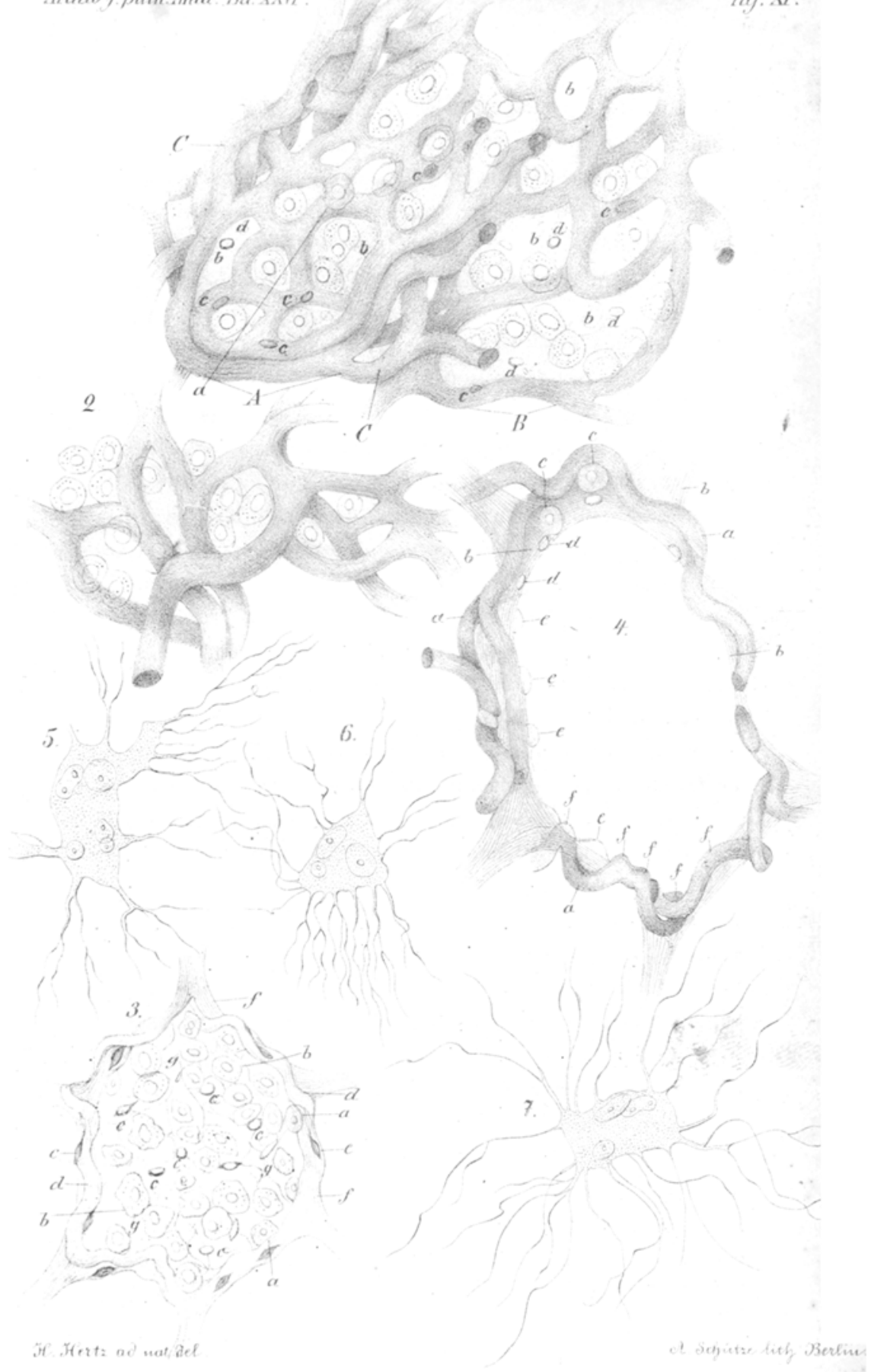

